Proyecciones Journal of Mathematics

Vol. 37, $\mathrm{N}^{o}$ 1, pp. 119-131, March 2018.

Universidad Católica del Norte

Antofagasta - Chile

\title{
Generalized Drazin-type spectra of Operator matrices
}

\author{
A. Tajmouati \\ Sidi Mohamed Ben Abdellah University, Maroc \\ M. Abkari \\ Sidi Mohamed Ben Abdellah University, Maroc \\ and \\ M. Karmouni \\ Cadi Ayyad University, Maroc \\ Received: May 2017. Accepted : August 2017
}

\begin{abstract}
In this paper, we investigate the limit points set of surjective and approximate point spectra of upper triangular operator matrices $M_{C}=$ $\left(\begin{array}{cc}A & C \\ 0 & B\end{array}\right)$. We prove that $\sigma_{*}\left(M_{C}\right) \cup W=\sigma_{*}(A) \cup \sigma_{*}(B)$ where $W$ is the union of certain holes in $\sigma_{*}\left(M_{C}\right)$, which happen to be subsets of $\sigma_{l g D}(B) \cap \sigma_{r g D}(A), \sigma_{*} \in\left\{\sigma_{l g D}, \sigma_{r g D}\right\}$ are the limit points set of surjective and approximate point spectra. Furthermore, several sufficient conditions for $\sigma_{*}\left(M_{C}\right)=\sigma_{*}(A) \cup \sigma_{*}(B)$ holds for every $C \in \mathcal{B}(Y, X)$ are given.
\end{abstract}

Subjclass [2010]: 47A10, 47A11.

Keywords : Surjective spectrum, approximate point spectrum, generalized Drazin spectrum, Single-valued extension property, operator matrices. 


\section{Introduction and Preliminaries}

Let $X, Y$ be Banach space and $\mathcal{B}(X, Y)$ denote the algebra of all bounded linear operators from $X$ to $Y$. For $Y=X$ we write $\mathcal{B}(X, X)=\mathcal{B}(X)$. For $T \in \mathcal{B}(X)$, we denote by $T^{*}, N(T), R(T), R^{\infty}(T)=\bigcap_{n>0} R\left(T^{n}\right), \sigma_{a p}(T)$, $\sigma_{s u}(T), \sigma_{p}(T), \rho(T)$ and $\sigma(T)$, respectively the adjoint, the null space, the range, the hyper-range, the approximate point spectrum, the surjectivity spectrum, the point spectrum, the resolvent set and the spectrum of $T$.

Next, let $T \in \mathcal{B}(X), T$ has the single valued extension property at $\lambda_{0} \in \mathbf{C}(\mathrm{SVEP})$ if for every open neighborhood $U \subseteq \mathbf{C}$ of $\lambda_{0}$, the only analytic function $f: U \longrightarrow X$ which satisfies the equation $(T-z I) f(z)=0$ for all $z \in U$ is the function $f \equiv 0$. T is said to have the SVEP if $T$ has the SVEP for every $\lambda \in \mathbf{C}$. Obviously, every operator $T \in \mathcal{B}(X)$ has the SVEP at every $\lambda \in \rho(T)$, then $T$ and $T^{*}$ have the SVEP at every point of the boundary $\partial(\sigma(T))$ of the spectrum. In particular, $T$ and $T^{*}$ have the SVEP at every isolated point of the spectrum. We denote by $S(T)$ the open set of $\lambda \in \mathbf{C}$ where $T$ fails to have SVEP at $\lambda$, and we say that $T$ has SVEP if $S(T)=\emptyset$. Note that $S(T) \subset \sigma_{p}(T)$ and $\sigma(T)=S(T) \cup \sigma_{s u}(T)$ see $[1,4]$.

For a compact subset $K$ of $\mathbf{C}$, let $a c c K$, int $K, i s o K, \partial K$ and $\eta(K)$ be the set of all points of accumulation of $K$, the interior of $K$, the isolated points of $K$, the boundary of $K$ and the polynomially convex hull of $K$ respectively.

Let $T \in \mathcal{B}(X), T$ is said to be Drazin invertible if there exists a positive integer $k$ and an operator $S \in \mathcal{B}(X)$ such that

$$
S T=T S, T^{k+1} S=T^{k} \text { and } S^{2} T=S .
$$

Which is also equivalent to the fact that $T=T_{1} \oplus T_{2}$; where $T_{1}$ is invertible and $T_{2}$ is nilpotent. The concept of Drazin invertible operators has been generalized by Koliha [5]. In fact, $T \in \mathcal{B}(X)$ is generalized Drazin invertible if and only if $0 \notin \operatorname{acc}(\sigma(T))$, which is also equivalent to the fact that $T=T_{1} \oplus T_{2}$ where $T_{1}$ is invertible and $T_{2}$ is quasi-nilpotent. The generalized Drazin invertible spectrum is defined by

$$
\sigma_{g D}(T)=\{\lambda \in \mathbf{C}: T-\lambda I \text { is not generalized Drazin invertible }\}
$$

Now, set

$$
\sigma_{l g D}(T)=\operatorname{acc}\left(\sigma_{a p}(T)\right)
$$




$$
\sigma_{r g D}(T)=\operatorname{acc}\left(\sigma_{s u}(T)\right) .
$$

Hence $\sigma_{g D}(T)=\sigma_{l g D}(T) \cup \sigma_{r g D}(T)$.

For $A \in \mathcal{B}(X), B \in \mathcal{B}(Y)$, we denote by $M_{C} \in \mathcal{B}(X \oplus Y)$ the operator defined on $X \oplus Y$ by

$$
\left(\begin{array}{cc}
A & C \\
0 & B
\end{array}\right)
$$

It is well known that, in the case of infinite dimensional, the inclusion $\sigma\left(M_{C}\right) \subset \sigma(A) \cup \sigma(B)$, may be strict. This motivates serval authors to study the defect set $\left(\sigma_{*}(A) \cup \sigma_{*}(B)\right) \backslash \sigma_{*}\left(M_{C}\right)$ where $\sigma_{*}$ runs different type spectra [2], [3], [6], [7], [9].

The following questions arise naturally:

1. Under which conditions on $A$ and $B$ does $\sigma_{*}\left(M_{C}\right)=\sigma_{*}(A) \cup \sigma_{*}(B)$ for arbitrary $C \in \mathcal{B}(Y, X)$ ?

2. Given $A$ and $B$, for which operators $C \in \mathcal{B}(Y, X)$ does $\sigma_{*}\left(M_{C}\right)=$ $\sigma_{*}(A) \cup \sigma_{*}(B) ?$

3. How to describe the passage of $\sigma_{*}\left(M_{0}\right)$ to $\sigma_{*}\left(M_{C}\right)$ ?

Where $\sigma_{*} \in\left\{\sigma_{l g D}, \sigma_{r g D}\right\}$.

In this paper, we motivated by the relationship between $\sigma_{*}\left(M_{C}\right)$ and $\sigma_{*}(A) \cup \sigma_{*}(B)$, where $\sigma_{*} \in\left\{\sigma_{l g D}, \sigma_{r g D}\right\}$. In addition, we show that the passage from $\sigma_{*}\left(M_{0}\right)$ to $\sigma_{*}\left(M_{C}\right)$ can be described as follows:

$$
\sigma_{*}\left(M_{C}\right) \cup W=\sigma_{*}\left(M_{0}\right)=\sigma_{*}(A) \cup \sigma_{*}(B)
$$

where $W$ is the union of certain holes in $\sigma_{*}\left(M_{C}\right)$, which happen to be subsets of $\sigma_{l g D}(B) \cap \sigma_{r g D}(A), \sigma_{*} \in\left\{\sigma_{l g D}, \sigma_{r g D}\right\}$.

\section{Main results}

We start this section by the following proposition.

Proposition 2.1. Let $(A, B) \in \mathcal{B}(X) \times \mathcal{B}(Y)$ and $C \in \mathcal{B}(Y, X)$. Then :

$$
\begin{gathered}
\sigma_{l g D}\left(M_{0}\right)=\sigma_{l g D}(A) \cup \sigma_{l g D}(B) \\
\sigma_{r g D}\left(M_{0}\right)=\sigma_{r g D}(A) \cup \sigma_{r g D}(B)
\end{gathered}
$$


Proof. $\quad \lambda \in \sigma_{l g D}\left(M_{0}\right)$ if and only if $\lambda \in \operatorname{acc}\left(\sigma_{a p}\left(M_{0}\right)\right)$ if and only if $\lambda \in \operatorname{acc}\left(\sigma_{a p}(A) \cup \sigma_{a p}(B)\right)=\operatorname{acc}\left(\sigma_{a p}(A)\right) \cup \operatorname{acc}\left(\sigma_{a p}(B)\right)$ if and only if $\lambda \in \sigma_{l g D}(A) \cup \sigma_{l g D}(B)$.

By duality, we have: $\sigma_{r g D}\left(M_{0}\right)=\sigma_{r g D}(A) \cup \sigma_{r g D}(B)$

As a straightforward consequence, we have the result of H.Zariouh and H. Zguitti [6].

Corollary 2.1. [6] Let $(A, B) \in \mathcal{B}(X) \times \mathcal{B}(Y)$ and $C \in \mathcal{B}(Y, X)$. Then :

$$
\sigma_{g D}\left(M_{0}\right)=\sigma_{g D}(A) \cup \sigma_{g D}(B)
$$

Theorem 2.1. Let $(A, B) \in \mathcal{B}(X) \times \mathcal{B}(Y)$ and $C \in \mathcal{B}(Y, X)$. Then :

$$
\begin{gathered}
\sigma_{l g D}(A) \subseteq \sigma_{l g D}\left(M_{C}\right) \subseteq \sigma_{l g D}(A) \cup \sigma_{l g D}(B) \subseteq \sigma_{l g D}\left(M_{C}\right) \cup \sigma_{l g D}(B) \\
\sigma_{r g D}(B) \subseteq \sigma_{r g D}\left(M_{C}\right) \subseteq \sigma_{r g D}(A) \cup \sigma_{r g D}(B) \subseteq \sigma_{r g D}\left(M_{C}\right) \cup \sigma_{r g D}(A)
\end{gathered}
$$

Proof. Without loss of generality, let $\mu=0 \notin \sigma_{l g D}(A) \cup \sigma_{l g D}(B)$, then $0 \notin a c c\left(\sigma_{a p}(A)\right) \cup a c c\left(\sigma_{a p}(B)\right)$. Thus there exists $\varepsilon>0$ such that for any $\lambda, 0<|\lambda|<\varepsilon$, we have $A-\lambda I$ and $B-\lambda I$ are bounded below. According to [7, Theorem 3.5], we have $M_{C}-\lambda I$ is bounded below for any $\lambda, 0<|\lambda|<\varepsilon$, thus $0 \notin \operatorname{acc}\left(\sigma_{a p}\left(M_{C}\right)\right)=\sigma_{l g D}\left(M_{C}\right)$. Therefore $\sigma_{l g D}\left(M_{C}\right) \subseteq \sigma_{l g D}(A) \cup \sigma_{l g D}(B)$.

If $0 \notin \sigma_{l g D}\left(M_{C}\right)$, then $0 \notin a c c\left(\sigma_{a p}\left(M_{C}\right)\right)$, as a result, there exists $\varepsilon>0$ such that for any $\lambda, 0<|\lambda|<\varepsilon$, we have $M_{C}-\lambda I$ is bounded below, then $A-\lambda I$ is bounded below for any $\lambda, 0<|\lambda|<\varepsilon$ by [7, Theorem 3.5], thus $0 \notin \sigma_{l g D}(A)$. Therefore $\sigma_{l g D}(A) \subseteq \sigma_{l g D}\left(M_{C}\right)$

Let $\mu=0 \notin \sigma_{l g D}\left(M_{C}\right) \cup \sigma_{l g D}(B)$, then $0 \notin a c c\left(\sigma_{a p}\left(M_{C}\right)\right) \cup a c c\left(\sigma_{a p}(B)\right)$. Thus there exists $\varepsilon>0$ such that for any $\lambda, 0<|\lambda|<\varepsilon$, we have $M_{C}-\lambda I$ and $B-\lambda I$ are bounded below. According to [7, Theorem 3.5], we have $A-\lambda I$ is bounded below for any $\lambda, 0<|\lambda|<\varepsilon$, thus $0 \notin \operatorname{acc}\left(\sigma_{a p}(A)\right)=$ $\sigma_{l g D}(A)$. Therefore $\sigma_{l g D}(A) \cup \sigma_{l g D}(B) \subseteq \sigma_{l g D}\left(M_{C}\right) \cup \sigma_{l g D}(B)$.

By duality, we have:

$\sigma_{r g D}(B) \subseteq \sigma_{r g D}\left(M_{C}\right) \subseteq \sigma_{r g D}\left(M_{0}\right)=\sigma_{r g D}(A) \cup \sigma_{r g D}(B) \subseteq \sigma_{r g D}\left(M_{C}\right) \cup \sigma_{r g D}(A)$

The inclusion, $\sigma_{r g D}\left(M_{C}\right) \subseteq \sigma_{r g D}(A) \cup \sigma_{r g D}(B)$, may be strict as we can see in the following example. 
Example 1. Let $A, B, C \in \mathcal{B}\left(l^{2}\right)$ defined by:

$$
\begin{gathered}
A e_{n}=e_{n+1} . \\
B=A^{*} . \\
C=e_{0} \otimes e_{0} .
\end{gathered}
$$

where $\left\{e_{n}\right\}_{n \in \mathbf{N}}$ is the unit vector basis of $l^{2}$. We have $\sigma_{s u}(A)=\{\lambda \in$ $\mathbf{C} ;|\lambda| \leq 1\}$, then $\sigma_{r g D}(A)=\{\lambda \in \mathbf{C} ;|\lambda| \leq 1\}$. Since $M_{C}$ is unitary, then $\sigma_{r g D}\left(M_{C}\right) \subseteq\{\lambda \in \mathbf{C} ;|\lambda|=1\}$. So $0 \notin \sigma_{r g D}\left(M_{C}\right)$, but $0 \in \sigma_{r g D}(A) \cup$ $\sigma_{r g D}(B)$. Notes that $A^{*}=B$ has not the SVEP. Also, we can show that the inclusion $\sigma_{l g D}\left(M_{C}\right) \subset \sigma_{l g D}(A) \cup \sigma_{l g D}(B)$ is strict. This will lead us to a necessary condition that ensures the equality desired.

The following proposition will be needed in the sequel.

Proposition 2.2. Let $(A, B) \in \mathcal{B}(X) \times \mathcal{B}(Y)$ and $C \in \mathcal{B}(Y, X)$.

1. If $A$ is invertible, then $0 \notin \sigma_{l g D}\left(M_{C}\right)$ if and only if $0 \notin \sigma_{l g D}(B)$.

2. If $B$ is invertible, then $0 \notin \sigma_{r g D}\left(M_{C}\right)$ if and only if $0 \notin \sigma_{r g D}(A)$.

Proof. 1) Suppose that $0 \notin \sigma_{l g D}\left(M_{C}\right), 0 \notin a c c\left(\sigma_{a p}\left(M_{C}\right)\right)$, then there exists $\varepsilon>0$ such that $M_{C}-\lambda I$ is bounded below for every $\lambda, 0<|\lambda|<\varepsilon$. Since $A$ is invertible, then there exists $\beta>0$ such that $A-\lambda I$ is invertible for every $\lambda,|\lambda|<\beta$. Let $\eta=\min (\varepsilon, \beta), A-\lambda I$ is invertible for every $\lambda,|\lambda|<\eta$ and $M_{C}-\lambda I$ is bounded below for every $\lambda, 0<|\lambda|<\eta$. Hence $B-\lambda I$ is bounded below for every $\lambda, 0<|\lambda|<\eta$, by [9, Lemma 2.7], the converse is similar.

By duality, we have 2).

Theorem 2.2. Let $(A, B) \in \mathcal{B}(X) \times \mathcal{B}(Y)$ and $C \in \mathcal{B}(Y, X)$. Then :

$$
\sigma_{l g D}\left(M_{C}\right) \cup S\left(A^{*}\right)=\sigma_{l g D}(A) \cup \sigma_{l g D}(B) \cup S\left(A^{*}\right)
$$

Proof. $\quad$ Since $\sigma_{l g D}\left(M_{C}\right) \subseteq \sigma_{l g D}(A) \cup \sigma_{l g D}(B)$, then $\sigma_{l g D}\left(M_{C}\right) \cup S\left(A^{*}\right) \subseteq$ $\sigma_{l g D}(A) \cup \sigma_{l g D}(B) \cup S\left(A^{*}\right)$. Conversely, let $\lambda \in\left(\sigma_{l g D}(A) \cup \sigma_{l g D}(B)\right) \backslash \sigma_{l g D}\left(M_{C}\right)$, we can assume without loss of generality that $\lambda=0$. Then $0 \notin \operatorname{acc}\left(\sigma_{a p}\left(M_{C}\right)\right)$, since $\sigma_{l g D}(A) \subseteq \sigma_{l g D}\left(M_{C}\right)$, then $0 \notin a c c\left(\sigma_{a p}(A)\right)$. Suppose that $0 \notin S\left(A^{*}\right)$ :

- If $0 \in \sigma(A)$, since $\sigma(A)=\sigma_{a p}(A) \cup S\left(A^{*}\right)$ then $0 \in \sigma_{a p}(A)$. As $0 \notin$ $\operatorname{acc}\left(\sigma_{a p}(A)\right)$ then $0 \in i s o\left(\sigma_{a p}(A)\right)$, therefore $0 \in i s o(\sigma(A))$, which implies 
that there exists $\varepsilon>0$ such that $\lambda I-A$ is invertible for all $\lambda, 0<|\lambda|<\varepsilon$, since $0 \notin a c c\left(\sigma_{a p}\left(M_{C}\right)\right)$, then there exists $\beta>0$ such that $\lambda-M_{C}$ is bounded below for all $0<|\lambda|<\beta$. Let $\alpha=\min (\beta, \varepsilon)$, then $\lambda I-A$ is invertible and $\lambda I-M_{C}$ is bounded below for all $0<|\lambda|<\alpha$, by $[9$, Lemma 2.7], we have $\lambda I-B$ is bounded below for all $\lambda, 0<|\lambda|<\alpha$, hence $0 \notin \operatorname{acc}\left(\sigma_{a p}(B)\right)$, thus $0 \notin \sigma_{l g D}(B)$. Then we have $0 \notin \sigma_{l g D}(A) \cup \sigma_{l g D}(B)$, contradiction.

- If $0 \notin \sigma(A)$ then $A$ is invertible and since $0 \notin \sigma_{l g D}\left(M_{C}\right)$, according to Proposition 2.2, we have $0 \notin \sigma_{l g D}(B)$, thus $0 \notin \sigma_{l g D}(A) \cup \sigma_{l g D}(B)$, contradiction.

Then $\left(\sigma_{l g D}(A) \cup \sigma_{l g D}(B)\right) \backslash \sigma_{l g D}\left(M_{C}\right) \subseteq S\left(A^{*}\right)$, this finishes the proof.

Theorem 2.3. Let $(A, B) \in \mathcal{B}(X) \times \mathcal{B}(Y)$ and $C \in \mathcal{B}(Y, X)$. Then :

$$
\sigma_{r g D}\left(M_{C}\right) \cup S(B)=\sigma_{r g D}(A) \cup \sigma_{r g D}(B) \cup S(B)
$$

Proof. $\quad$ Since $\sigma_{r g D}\left(M_{C}\right) \subseteq \sigma_{r g D}(A) \cup \sigma_{r g D}(B)$, then $\sigma_{r g D}\left(M_{C}\right) \cup S(B) \subseteq$ $\sigma_{r g D}(A) \cup \sigma_{r g D}(B) \cup S(B)$. Conversely, let $\lambda \in\left(\sigma_{r g D}(A) \cup \sigma_{r g D}(B)\right) \backslash \sigma_{r g D}\left(M_{C}\right)$, we can assume without loss of generality that $\lambda=0$. Then $0 \notin a c c\left(\sigma_{s u}\left(M_{C}\right)\right)$, since $\sigma_{r g D}(B) \subseteq \sigma_{r g D}\left(M_{C}\right)$, then $0 \notin a c c\left(\sigma_{s u}(B)\right)$. Suppose that $0 \notin S(B)$ :

- If $0 \in \sigma(B)$, since $\sigma(B)=\sigma_{s u}(B) \cup S(B)$, then $0 \in \sigma_{s u}(B)$. As $0 \notin$ $\operatorname{acc}\left(\sigma_{s u}(B)\right)$ then $0 \in i s o\left(\sigma_{s u}(B)\right)$, therefore $0 \in i s o(\sigma(B))$, which implies that there exists $\varepsilon>0$ such that $\lambda I-B$ is invertible for all $\lambda, 0<|\lambda|<\varepsilon$, since $0 \notin \operatorname{acc}\left(\sigma_{s u}\left(M_{C}\right)\right)$, then there exists $\beta>0$ such that $\lambda I-M_{C}$ is surjective for all $\lambda, 0<|\lambda|<\beta$. Let $\alpha=\min (\beta, \varepsilon)$, then $\lambda I-B$ is invertible and $\lambda I-M_{C}$ is surjective for all $\lambda, 0<|\lambda|<\alpha$, by [9, Lemma 2.7], we have $\lambda I-A$ is surjective for all $\lambda, 0<|\lambda|<\alpha$, hence $0 \notin \operatorname{acc}\left(\sigma_{s u}(A)\right)$, thus $0 \notin \sigma_{r g D}(A)$. Then we have $0 \notin \sigma_{r g D}(A) \cup \sigma_{r g D}(B)$, contradiction.

- If $0 \notin \sigma(B)$ then $B$ is invertible and since $0 \notin \sigma_{r g D}\left(M_{C}\right)$, according to Proposition 2.2, we have $0 \notin \sigma_{r g D}(A)$, thus $0 \notin \sigma_{r g D}(A) \cup \sigma_{r g D}(B)$, contradiction.

Corollary 2.2. 1) Let $A \in \mathcal{B}(X)$. If $S\left(A^{*}\right)=\emptyset$, then for every $B \in \mathcal{B}(Y)$ and $C \in \mathcal{B}(Y, X)$, we have:

$$
\sigma_{l g D}\left(M_{C}\right)=\sigma_{l g D}(A) \cup \sigma_{l g D}(B)
$$


2) Let $B \in \mathcal{B}(Y)$. If $S(B)=\emptyset$ then for every $A \in \mathcal{B}(X)$ and $C \in \mathcal{B}(Y, X)$, we have:

$$
\sigma_{r g D}\left(M_{C}\right)=\sigma_{r g D}(A) \cup \sigma_{r g D}(B)
$$

Lemma 2.1. Let $T \in \mathcal{B}(X)$. Then:

$$
S(T) \subset \sigma_{l g D}(T) \text { and } S\left(T^{*}\right) \subset \sigma_{r g D}(T) \text {. }
$$

Corollary 2.3. Let $(A, B) \in \mathcal{B}(X) \times \mathcal{B}(Y)$ and $C \in \mathcal{B}(Y, X)$. If one of the following conditions holds:

1. $\operatorname{int}\left(\sigma_{p}\left(A^{*}\right)\right)=\emptyset$,

2. $\operatorname{int}\left(\sigma_{r g D}(A)\right)=\emptyset$,

3. $\sigma_{l g D}(B) \cap \sigma_{r g D}(A)=\emptyset$.

then we have

$$
\sigma_{l g D}\left(M_{C}\right)=\sigma_{l g D}(A) \cup \sigma_{l g D}(B)
$$

Proof. $\quad$ Note that $S\left(A^{*}\right) \subseteq \sigma_{p}\left(A^{*}\right)$. If $\operatorname{int}\left(\sigma_{p}\left(A^{*}\right)\right)=\emptyset$, by Corollary 2.2, we have $\sigma_{l g D}\left(M_{C}\right)=\sigma_{l g D}(A) \cup \sigma_{l g D}(B)$.

If $\operatorname{int}\left(\sigma_{r g D}(A)\right)=\emptyset$, as $S\left(A^{*}\right) \subseteq \sigma_{r g D}(A)$, by Corollary 2.2 we have the result.

According to Theorem 2.1, we have $\left(\sigma_{l g D}(A) \cup \sigma_{l g D}(B)\right) \backslash \sigma_{l g D}\left(M_{C}\right) \subseteq$ $\sigma_{l g D}(B)$ and from Theorem $2.2\left(\sigma_{l g D}(A) \cup \sigma_{l g D}(B)\right) \backslash \sigma_{l g D}\left(M_{C}\right) \subseteq S\left(A^{*}\right)$, since $S\left(A^{*}\right) \subseteq \sigma_{r g D}(A)$, then $\left(\sigma_{l g D}(A) \cup \sigma_{l g D}(B)\right) \backslash \sigma_{l g D}\left(M_{C}\right) \subseteq \sigma_{l g D}(B) \cap$ $\sigma_{r g D}(A)$. If $\sigma_{l g D}(B) \cap \sigma_{r g D}(A)=\emptyset$, then $\sigma_{l g D}\left(M_{C}\right)=\sigma_{l g D}(A) \cup \sigma_{l g D}(B)$.

Corollary 2.4. Let $(A, B) \in \mathcal{B}(X) \times \mathcal{B}(Y)$ and $C \in \mathcal{B}(Y, X)$. If one of the following conditions holds:

1. $\operatorname{int}\left(\sigma_{p}(B)\right)=\emptyset$,

2. $\operatorname{int}\left(\sigma_{l g D}(B)\right)=\emptyset$,

3. $\sigma_{l g D}(B) \cap \sigma_{r g D}(A)=\emptyset$.

then we have

$$
\sigma_{r g D}\left(M_{C}\right)=\sigma_{r g D}(A) \cup \sigma_{r g D}(B)
$$


Proof. $\quad$ Note that $S(B) \subseteq \sigma_{p}(B)$. If $\operatorname{int}\left(\sigma_{p}(B)\right)=\emptyset$, by Corollary 2.2, we have $\sigma_{r g D}\left(M_{C}\right)=\sigma_{r g D}(A) \cup \sigma_{r g D}(B)$.

If $\operatorname{int}\left(\sigma_{l g D}(B)\right)=\emptyset$, as $S(B) \subseteq \sigma_{l g D}(B)$, by Corollary 2.2 we have the result.

From Theorem 2.1, we have $\left(\sigma_{r g D}(A) \cup \sigma_{r g D}(B)\right) \backslash \sigma_{r g D}\left(M_{C}\right) \subseteq \sigma_{r g D}(A)$ and from Theorem $2.3\left(\sigma_{r g D}(A) \cup \sigma_{r g D}(B)\right) \backslash \sigma_{r g D}\left(M_{C}\right) \subseteq S(B)$, since $S(B) \subseteq$ $\sigma_{l g D}(B)$, then $\left(\sigma_{r g D}(A) \cup \sigma_{r g D}(B)\right) \backslash \sigma_{r g D}\left(M_{C}\right) \subseteq \sigma_{l g D}(B) \cap \sigma_{r g D}(A)$. If $\sigma_{l g D}(B) \cap \sigma_{r g D}(A)=\emptyset$, then $\sigma_{l g D}\left(M_{C}\right)=\sigma_{l g D}(A) \cup \sigma_{l g D}(B)$.

For $(A, B) \in \mathcal{B}(X) \times \mathcal{B}(Y)$, let $L_{A}$ (resp. $\left.R_{B}\right)$ be the left (resp. right) multiplication operator given by $L_{A}(X)=A X ;\left(\right.$ resp. $\left.R_{B}(X)=X B\right)$, and let $\delta_{A, B}(X)=A X-X B=L_{A}(X)-R_{B}(X)$ be the usual generalized derivation associated with $A$ and $B$. When $\mathrm{A}=\mathrm{B}$, we simply write $\delta_{A, A}=$ $\delta_{A} \cdot N^{\infty}(A)=\bigcup_{n \geq 1} N\left(A^{n}\right)$ the generalized kernel of $A$.

The following theorem gives an answer to Question 2.

Theorem 2.4. Let $A \in \mathcal{B}(X)$ and $B \in \mathcal{B}(Y)$. If $C$ is in the closure of the set

$$
R\left(\delta_{A, B}\right)+N\left(\delta_{A, B}\right)+\bigcup_{\lambda, \mu \in \mathbf{C}}\left[N^{\infty}\left(L_{A-\lambda}\right) \cap N^{\infty}\left(R_{B-\mu}\right)\right]
$$

then :

$$
\begin{gathered}
\sigma_{l g D}\left(M_{0}\right)=\sigma_{l g D}\left(M_{C}\right) \\
\sigma_{r g D}\left(M_{0}\right)=\sigma_{r g D}\left(M_{C}\right)
\end{gathered}
$$

Proof. If $C$ is in the closure of the set

$$
R\left(\delta_{A, B}\right)+N\left(\delta_{A, B}\right)+\bigcup_{\lambda, \mu \in \mathbf{C}}\left[N^{\infty}\left(L_{A-\lambda}\right) \cap N^{\infty}\left(R_{B-\mu}\right)\right]
$$

then, $\sigma_{a p}\left(M_{C}\right) \backslash\{0\}=\sigma_{a p}\left(M_{0}\right) \backslash\{0\}$ and $\sigma_{s u}\left(M_{C}\right) \backslash\{0\}=\sigma_{s u}\left(M_{0}\right) \backslash\{0\}$, see [2, Theorem 2.2], it remains to discuss the cas of the origin. Let $C \in$ $N^{\infty}\left(L_{A-\lambda}\right) \cap N^{\infty}\left(R_{B-\mu}\right)$, because of translation stability of spectra, we may assume $\lambda=\mu=0$. If $C \in N\left(L_{A}^{n}\right)$ is a nonzero operator, then $0 \in$ $\sigma_{p}(A) \subseteq \sigma_{a p}(A) \subseteq \sigma_{a p}\left(M_{C}\right) \cap \sigma_{a p}\left(M_{0}\right)$. On the other hand by duality, we use the assumption $C \in N\left(R_{B}^{n}\right)$ to obtain $0 \in \sigma_{s u}(B) \subseteq \sigma_{s u}\left(M_{C}\right) \cap \sigma_{s u}\left(M_{0}\right)$. Finally $\sigma_{a p}\left(M_{C}\right)=\sigma_{a p}\left(M_{0}\right)$ and $\sigma_{s u}\left(M_{C}\right)=\sigma_{s u}\left(M_{0}\right)$.

Remark 1. 1) Let $T$ defined on $l^{2}(\mathbf{N})$ by $T\left(x_{1}, x_{2}, \ldots\right)=\left(0, x_{1}, x_{2}, \ldots\right)$. Let $A=T, B=T^{*}$ and $C=A-B$, then $C \in R\left(\delta_{A, B}\right)$, according to theorem 2.4, we have $\sigma_{l g D}\left(M_{0}\right)=\sigma_{l g D}\left(M_{C}\right)$ and $\sigma_{r g D}\left(M_{0}\right)=\sigma_{r g D}\left(M_{C}\right)$. But, $S\left(A^{*}\right)=S(B)=\{\lambda \in \mathbf{C},|\lambda|<1\}$. We conclude that in general there is no 
definite relation between the condition considered in corollary 2.2 and the condition considered in the above theorem.

2) The closure of the set $R\left(\delta_{A, B}\right)+N\left(\delta_{A, B}\right)+\bigcup_{\lambda, \mu \in \mathbf{C}}\left[N^{\infty}\left(L_{A-\lambda}\right) \cap N^{\infty}\left(R_{B-\mu}\right)\right]$ is not the largest class for which $\sigma_{l g D}\left(M_{0}\right)=\sigma_{l g D}\left(M_{C}\right)$ and $\sigma_{r g D}\left(M_{0}\right)=$ $\sigma_{r g D}\left(M_{C}\right)$ hold. Indeed: Let $A \in \mathcal{B}(X)$ such that $A^{3}=0 \neq A^{2}$ and $A=B$. For every $C \in \mathcal{B}(X)$, we have $\sigma_{l g D}\left(M_{0}\right)=\sigma_{l g D}\left(M_{C}\right)$ and $\sigma_{r g D}\left(M_{0}\right)=\sigma_{r g D}\left(M_{C}\right)$. A simple computation shows that $\delta_{A}^{5}=0$ then $R\left(\delta_{A}\right) \subseteq N\left(\delta_{A}^{4}\right)$. If $\lambda \in \mathbf{C}, C \in N\left(L_{(A-\lambda)^{2}}\right)$, then $\left(\lambda^{2}-2 \lambda A+A^{2}\right) C=0$. Since $A^{3}=0$, we conclude that $A^{2} C=0$ and so $C \in N\left(\delta_{A}^{4}\right)$. Then

$$
\bigcup_{\lambda, \mu \in \mathbf{C}}\left[N^{\infty}\left(L_{A-\lambda}\right) \cap N^{\infty}\left(R_{B-\mu}\right)\right] \subseteq N\left(\delta_{A}^{4}\right)
$$

Consequently, we have

$$
c l\left(R\left(\delta_{A}\right)+N\left(\delta_{A}\right)+\bigcup_{\lambda, \mu \in \mathbf{C}}\left[N^{\infty}\left(L_{A-\lambda}\right) \cap N^{\infty}\left(R_{B-\mu}\right)\right]\right) \subseteq N\left(\delta_{A}^{4}\right) \neq \mathcal{B}(X)
$$

In [9], it was shown that the passage from $\sigma_{g D}\left(M_{0}\right)$ to $\sigma_{g D}\left(M_{C}\right)$ is accomplished removing certain open subsets of $\sigma_{g D}(A) \cap \sigma_{g D}(B)$ from the former, that is, there is equality

$$
\sigma_{g D}(A) \cup \sigma_{g D}(B)=\sigma_{g D}\left(M_{0}\right)=\sigma_{g D}\left(M_{C}\right) \cup W
$$

where $W$ is the union of certain of the holes in $\sigma_{g D}\left(M_{C}\right)$ which happen to be subsets of $\sigma_{g D}(A) \cap \sigma_{g D}(B)$. The passage from $\sigma_{l g D}\left(M_{0}\right)$ (resp. $\left.\sigma_{r g D}\left(M_{0}\right)\right)$ to $\sigma_{l g D}\left(M_{C}\right)$ (resp. $\left.\sigma_{r g D}\left(M_{C}\right)\right)$ is more delicate.

Theorem 2.5. Let $(A, B) \in \mathcal{B}(X) \times \mathcal{B}(Y)$ and $C \in \mathcal{B}(Y, X)$. Then

$$
\sigma_{l g D}\left(M_{C}\right) \cup W=\sigma_{l g D}(A) \cup \sigma_{l g D}(B)
$$

where $W$ is the union of certain holes in $\sigma_{l g D}\left(M_{C}\right)$, which happen to be subsets of $\sigma_{l g D}(B) \cap \sigma_{r g D}(A)$.

And

$$
\sigma_{r g D}\left(M_{C}\right) \cup W^{\prime}=\sigma_{r g D}(A) \cup \sigma_{r g D}(B)
$$

where $W^{\prime}$ is the union of certain holes in $\sigma_{r g D}\left(M_{C}\right)$, which happen to be subsets of $\sigma_{l g D}(B) \cap \sigma_{r g D}(A)$. 
Proof. First, we can claim that, for every $C \in \mathcal{B}(Y, X)$.

$$
\begin{gathered}
\left(\sigma_{l g D}(A) \cup \sigma_{l g D}(B)\right) \backslash \sigma_{l g D}\left(M_{C}\right) \subseteq \sigma_{l g D}(B) \cap \sigma_{r g D}(A) \\
\sigma_{l g D}\left(M_{C}\right) \subseteq \sigma_{l g D}(A) \cup \sigma_{l g D}(B)
\end{gathered}
$$

Indeed, the second inclusion follows from Theorem 2.1. For the first inclusion, according to Theorem 2.1, we have $\left(\sigma_{l g D}(A) \cup \sigma_{l g D}(B)\right) \backslash \sigma_{l g D}\left(M_{C}\right) \subseteq$ $\sigma_{l g D}(B)$ and from Theorem $2.2\left(\sigma_{l g D}(A) \cup \sigma_{l g D}(B)\right) \backslash \sigma_{l g D}\left(M_{C}\right) \subseteq S\left(A^{*}\right)$, since $S\left(A^{*}\right) \subseteq \sigma_{r g D}(A)$, then $\left(\sigma_{l g D}(A) \cup \sigma_{l g D}(B)\right) \backslash \sigma_{l g D}\left(M_{C}\right) \subseteq \sigma_{l g D}(B) \cap$ $\sigma_{r g D}(A)$.

Next we claim that, for every $T \in \mathcal{B}(X)$, we have

$$
\eta\left(\sigma_{l g D}(T)\right)=\eta\left(\sigma_{g D}(T)\right)
$$

where $\eta(K)$ denotes the polynomially convex hull of the compact subset $K$ of $\mathbf{C}$.

Since $\sigma_{l g D}(T) \subseteq \sigma_{g D}(T)$, we need to prove $\partial \sigma_{g D}(T) \subseteq \partial \sigma_{l g D}(T)$. But since $\operatorname{int}\left(\sigma_{l g D}(T)\right) \subseteq \operatorname{int}\left(\sigma_{g D}(T)\right)$, it suffices to show that $\partial \sigma_{g D}(T) \subseteq$ $\sigma_{l g D}(T)$. Without loss of generality, suppose $0 \in \partial \sigma_{g D}(T)$. There are two cases to consider.

Case 1: If $0 \in \operatorname{acc}\left(\partial \sigma_{g D}(T)\right)$, then there exists $\left(\lambda_{n}\right) \subseteq \partial\left(\sigma_{g D}(T)\right)$, such that $\lim _{n \rightarrow \infty} \lambda_{n}=0$, since

$$
\partial\left(\sigma_{g D}(T)\right)=\partial(\operatorname{acc} \sigma(T)) \subseteq \operatorname{acc} \sigma(T) \backslash i n t \sigma(T) \subseteq \partial(\sigma(T)) \subseteq \sigma_{a p}(T)
$$

we have, $\lambda_{n} \in \sigma_{a p}(T), n=1,2, \ldots$, hence $0 \in \operatorname{acc}\left(\sigma_{a p}(T)\right)=\sigma_{l g D}(T)$.

Case 2: If $0 \in i s o\left(\partial \sigma_{g D}(T)\right)$, since $\sigma_{g D}(T)$ is closed, then $i s o\left(\partial \sigma_{g D}(T)\right)=$ $i s o\left(\sigma_{g D}(T)\right) .0 \in i s o\left(\sigma_{g D}(T)\right)=i s o(\operatorname{acc} \sigma(T))$, then $0 \in \operatorname{acc} \sigma(T)$ and there exists $\varepsilon>0$ such that $\lambda \notin \operatorname{acc}(\sigma(T))$ for every $\lambda, 0<|\lambda|<\varepsilon$. Since $0 \in \operatorname{acc} \sigma(T)$, there exists $\left(\mu_{n}\right) \subseteq \sigma(T)$ such that $\lim _{n \rightarrow \infty} \mu_{n}=0, \mu_{n} \neq 0$ for all $n$, thus there exists certain positive integer $N$ such that $0<\left|\mu_{n}\right|<\varepsilon$ for any $n \geq N$. Let $\lambda_{n}=\mu_{N+1+n}$, then $\lambda_{n} \in i \operatorname{so}(\sigma(T)) n=1,2, .$. and $\lim _{n \rightarrow \infty} \lambda_{n}=0$. Since $\sigma(T)$ is closed, then

$$
i s o(\sigma(T)) \subseteq \partial(\sigma(T)) \subseteq \sigma_{a p}(T)
$$


Then, $\lambda_{n} \in i s o(\sigma(T)) \subseteq \sigma_{a p}(T) n=1,2, \ldots$ Since $\lim _{n \rightarrow \infty} \lambda_{n}=0$, then $0 \in \operatorname{acc}\left(\sigma_{a p}(T)\right)$. So $0 \in \sigma_{l g D}(T)$.

Then $\partial \sigma_{g D}(T) \subseteq \sigma_{l g D}(T)$. This proves (2). Similarly, for every $T \in \mathcal{B}(X)$ and $S \in \mathcal{B}(Y), \eta\left(\sigma_{l g D}(T) \cup \sigma_{l g D}(S)\right)=\eta\left(\sigma_{g D}(T) \cup \sigma_{g D}(S)\right)$. From [8], if $(A, B) \in \mathcal{B}(X) \times \mathcal{B}(Y)$ and $C \in \mathcal{B}(Y, X)$, we have

$$
\eta\left(\sigma_{g D}\left(M_{C}\right)\right)=\eta\left(\sigma_{g D}(A) \cup \sigma_{g D}(B)\right)
$$

Then

$\eta\left(\sigma_{l g D}\left(M_{C}\right)\right)=\eta\left(\sigma_{g D}\left(M_{C}\right)\right)=\eta\left(\sigma_{g D}(A) \cup \sigma_{g D}(B)\right)=\eta\left(\sigma_{l g D}(A) \cup \sigma_{l g D}(B)\right)$

Hence

$$
\eta\left(\sigma_{l g D}\left(M_{C}\right)\right)=\eta\left(\sigma_{l g D}(A) \cup \sigma_{l g D}(B)\right)
$$

(3) says that the passage from $\sigma_{l g D}\left(M_{C}\right)$ to $\sigma_{l g D}(A) \cup \sigma_{l g D}(B)$ is the filling in certain of the holes in $\sigma_{l g D}\left(M_{C}\right)$. But since $\left(\sigma_{l g D}(A) \cup \sigma_{l g D}(B)\right) \backslash \sigma_{l g D}\left(M_{C}\right)$ is contained in $\sigma_{l g D}(B) \cap \sigma_{r g D}(A)$, it follows that the filling in certain of the holes in $\sigma_{l g D}\left(M_{C}\right)$ should occur in $\sigma_{l g D}(B) \cap \sigma_{r g D}(A)$.

Similarly we have 2)

Corollary 2.5. Let $(A, B) \in \mathcal{B}(X) \times \mathcal{B}(Y)$. If $\sigma_{l g D}(B) \cap \sigma_{\text {rgD }}(A)$ has no interior points, then for every $C \in \mathcal{B}(Y, X)$, we have

$$
\begin{aligned}
& \text { 1. } \sigma_{l g D}\left(M_{C}\right)=\sigma_{l g D}(A) \cup \sigma_{l g D}(B) \\
& \text { 2. } \sigma_{r g D}\left(M_{C}\right)=\sigma_{r g D}(A) \cup \sigma_{r g D}(B) \\
& \text { 3. } \sigma_{g D}\left(M_{C}\right)=\sigma_{g D}(A) \cup \sigma_{g D}(B)
\end{aligned}
$$

\section{Acknowledgements:}

We wish to thank the referee for his valuable comments and suggestions.

\section{References}

[1] P. Aiena. Fredholm and Local Spectral Theory with Applications to Multipliers. Kluwer. Acad. Press, (2004). 
[2] C. Benhida, E. H. Zerouali, H. Zguitti. Spectra of upper triangular operator matrices, Proc. Am. Math. Soc. Vol 133, Num 10, pp. 30133020, (2005).

[3] M. Houmidi, H. Zguitti, Propriétés spectrales locales d'une matrice carrée des opérateurs, Acta Math. Vietnam. 25, pp. 137-144, (2000).

[4] K. B.Laursen, M. M. Neumann. An introduction to Local Spectral Theory in: London Mathematical Society Monograph, New series, Vol. 20, Clarendon Press, Oxford, (2000).

[5] J. J. Koliha. A generalized Drazin inverse. Glasgow Math. J., 38 : pp. 367-81, (1996).

[6] H. Zariouh, H. Zguitti. On pseudo B-Weyl operators and generalized drazin invertible for operator matrices, Linear and Multilinear Algebra, Vol 64, Issue 7, pp. 1245-1257, (2016).

[7] E. H. Zerouali, H. Zguitti. Perturbation of spectra of operator matrices and local spectral theory, J. Math Anal Appl, 324: pp. 992-1005, (2006).

[8] S. Zhang, H.Zhong, L. Lin. Generalized Drazin Spectrum of Operator Matrices, Appl. Math. J. Chinese Univ. 29 (2), pp. 162-170, (2014).

[9] S. F. Zhang, H. J. Zhang, J. D. Wu. Spectra of upper-triangular operator matrices, Acta Math Sinica (in Chinese), 2011, 54: 41-60, (2011).

\section{A. Tajmouati}

Sidi Mohamed Ben Abdellah University

Faculty of Sciences Dhar Al Mahraz,

Laboratory of Mathematical Analysis and Applications,

Fez,

Morocco

e-mail : abdelaziz.tajmouati@usmba.ac.ma 


\section{Abkari}

Sidi Mohamed Ben Abdellah University

Faculty of Sciences Dhar Al Mahraz,

Laboratory of Mathematical Analysis and Applications,

Fez,

Morocco

e-mail : mbark.abkari@usmba.ac.ma

and

\section{Karmouni}

Cadi Ayyad University,

Multi-disciplinary Faculty of Safi, B. P. 4162 Sidi Bouzid Safi,

Morocco

e-mail : med89karmouni@gmail.com 